\title{
Virtual reality distraction decreases routine intravenous sedation and procedure-related pain during preoperative adductor canal catheter insertion: a retrospective study
}

\author{
Pooja G. Pandya ${ }^{1}$, T. Edward Kim ${ }^{1,2}$, Steven K. Howard ${ }^{1,2}$, Erica Stary ${ }^{1,2}$, \\ Jody C. Leng ${ }^{1,2}$, Oluwatobi O. Hunter ${ }^{2}$, and Edward R. Mariano ${ }^{1,2}$ \\ ${ }^{I}$ Department of Anesthesiology, Perioperative and Pain Medicine, Stanford University School of Medicine, \\ ${ }^{2}$ Anesthesiology and Perioperative Care Service, Veterans Affairs Palo Alto Health Care System, Palo Alto, CA, USA
}

\begin{abstract}
Background: Virtual reality (VR) distraction is a nonpharmacological method to prevent acute pain that has not yet been thoroughly explored for anesthesiology. We present our experience using VR distraction to decrease routine intravenous sedation for patients undergoing preoperative perineural catheter insertion.

Methods: This 1-month quality improvement project involved all elective unilateral primary total knee arthroplasty patients who received a preoperative adductor canal catheter. Clinical data were analyzed retrospectively. For the first half of the month, all patients received usual care; intravenous sedation was administered at the discretion of the regional anesthesiologist. For the second half of the month, patients were offered VR distraction with intravenous sedation upon request. The primary outcome was fentanyl dosage; other outcomes included midazolam dosage, procedure-related pain, procedural time, and blood pressure changes.

Results: Seven patients received usual care and seven used VR. In the VR group, 1/7 received intravenous sedation versus $6 / 7$ who received usual care $(\mathrm{P}=0.029)$. The fentanyl dose was lower (median [10th-90th percentiles]) in the VR group $(0[0-20] \mu \mathrm{g})$ versus the non-VR group $(50$ [30-100] $\mu \mathrm{g} ; \mathrm{P}=0.008)$. Midazolam use was lower in the VR group (0 [0-0] $\mathrm{mg}$ ) than in the non-VR group (1 [0-1] mg; $\mathrm{P}=0.024)$. Procedure-related pain was lower in the VR group (1 [1-4] NRS) versus the non-VR group (3 [2-6] NRS; $P=0.032$ ). There was no difference in other outcomes.

Conclusions: VR distraction may provide an effective nonpharmacological alternative to intravenous sedation for the ultrasound-guided placement of certain perineural catheters.
\end{abstract}

Key Words: Opioids, Perineural catheter, Quality improvement, Regional anesthesia, Ultrasound, Virtual reality.

Corresponding author: Edward R. Mariano, M.D., M.A.S.

Anesthesiology and Perioperative Care Service, Veterans Affairs Palo Alto Health Care System, 3801 Miranda Avenue (112A), Palo Alto, CA 94304, USA

Tel: 1-650-849-0254, Fax: 82-1-650-852-3423, Email: emariano@stanford.edu

ORCID: https://orcid.org/0000-0003-2735-248X

Received: December 30, 2016. Revised: January 20, 2017 (1st); February 7, 2017 (2nd). Accepted: February 7, 2017.

Korean J Anesthesiol 2017 August 70(4): 439-445

https://doi.org/10.4097/kjae.2017.70.4.439

(c) This is an open-access article distributed under the terms of the Creative Commons Attribution Non-Commercial License (http://creativecommons.org/ licenses/by-nc/4.0/), which permits unrestricted non-commercial use, distribution, and reproduction in any medium, provided the original work is properly cited. 


\section{Introduction}

Ultrasound use decreases pain related to perineural catheter insertion compared with traditional methods [1,2]. However, despite lower procedure-related pain scores, it is still common to administer intravenous sedation, including potent opioids like fentanyl [3] and benzodiazepines like midazolam, to achieve patient comfort. These medications are not benign. Opioids, in particular, should not be given routinely because side effects such as respiratory depression can be seen, especially in patients with sleep apnea [4], and even opioid-naïve patients who receive opioids in the perioperative period are at risk for long-term opioid use [5]. Furthermore, benzodiazepines and intravenous opioids may contribute to postoperative delirium in vulnerable patients, like the elderly [6].

Virtual reality (VR) distraction has been used on a limited basis as a nonpharmacological method to prevent acute pain related to burn dressing changes and other minor procedures $[7,8]$. Its utility in the perioperative period has not been explored thoroughly, and it has not been studied previously as an analgesic modality for anesthesiology procedures. Commercially available and inexpensive VR devices now make the technology much more accessible. Thus, we performed a quality improvement (QI) project using VR distraction with an intent to eliminate routine intravenous procedural sedation in patients undergoing preoperative adductor canal catheter (ACC) placement prior to elective total knee arthroplasty (TKA).

\section{Materials and Methods}

The local Veterans Affairs research committee and our Institutional Review Board approved this retrospective cohort study with a waiver of the need for informed consent. QI is a necessary and integral part of hospital operations, especially in a learning healthcare system, and the data included in our analyses were collected during the conduct of routine clinical care.

\section{Project design}

As an initial pilot, the scope of this QI project was limited to patients scheduled to undergo elective unilateral primary TKA who consented to have preoperative ACC insertion with a postoperative perineural local anesthetic infusion. VR distraction was implemented in this patient population for the following reasons: 1) our institution has an established institutional clinical pathway incorporating ultrasound-guided ACC insertion for all patients scheduled for TKA [9]; 2) the adductor canal location seemed ideal due to a lower extremity location that would not be disturbed by patient movement of the head and neck during a VR experience; and 3) all preoperative regional anesthesia procedures at our institution are performed in a designated block room equipped with standard monitors mandated by the American Society of Anesthesiologists, a supplemental oxygen source, and resuscitation supplies and medications immediately available. For the first half of the month, patients received usual care, which consisted of intravenous sedation offered and administered at the discretion of the regional anesthesiologist and titrated to patient comfort while ensuring patients' ability to answer questions and follow commands. For the second half of the month, patients were offered VR distraction and told that intravenous sedation was available and would be administered immediately upon request. The selection of patients to receive VR for this QI project was not random and was dependent on the availability of the VR distraction device and a practitioner to coach patients on its use.

\section{VR distraction technique}

Patients who were offered and who chose to try VR distraction were first oriented to the device set-up: Hypervision 2D Virtual Reality Glasses (CJ Global Inc., Fair Lawn, NJ, USA; Fig. 1) and disposable single-patient-use headphones (included in patient hospitality kits). Patients chose one of three free reality applications (Fig. 2) compatible with the Android platform to run on a Galaxy S4 Smartphone (Samsung, Seoul, South Korea): A) Titans of Space (Drash VR LLC, Murrieta, CA, USA), B) Lanterns for Google Cardboard (Oleksandr Popov, Svitlovodsk, Ukraine), or C) SeaWorld VR2 (eiNpictures, Incheon, South Korea). Patients were coached by a separate practitioner aside from the regional anesthesiologists performing the ACC procedure. All three applications are three-dimensional immersive VR experiences consisting of vivid scenes and background music. Titans of Space is the most interactive; the user takes a self-guided tour of our solar system and uses gaze fixation to open up information windows about each planet and move from one scene to

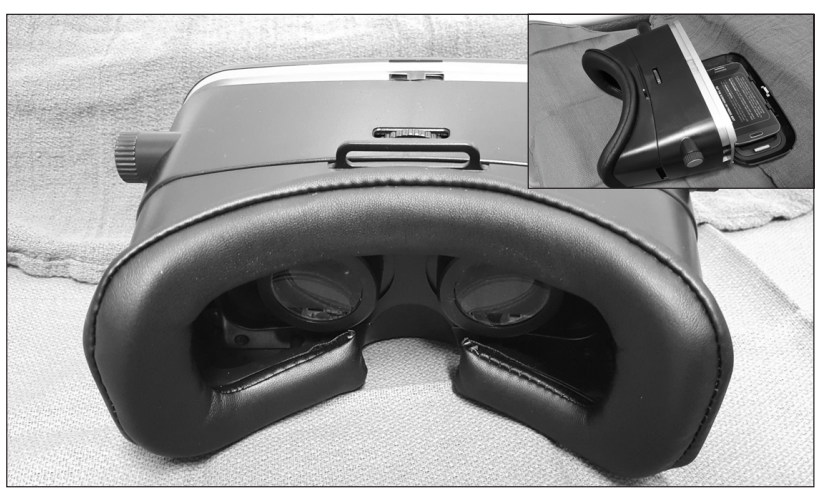

Fig. 1. Image showing the virtual reality interface used; the inset box shows the location of the smartphone. 

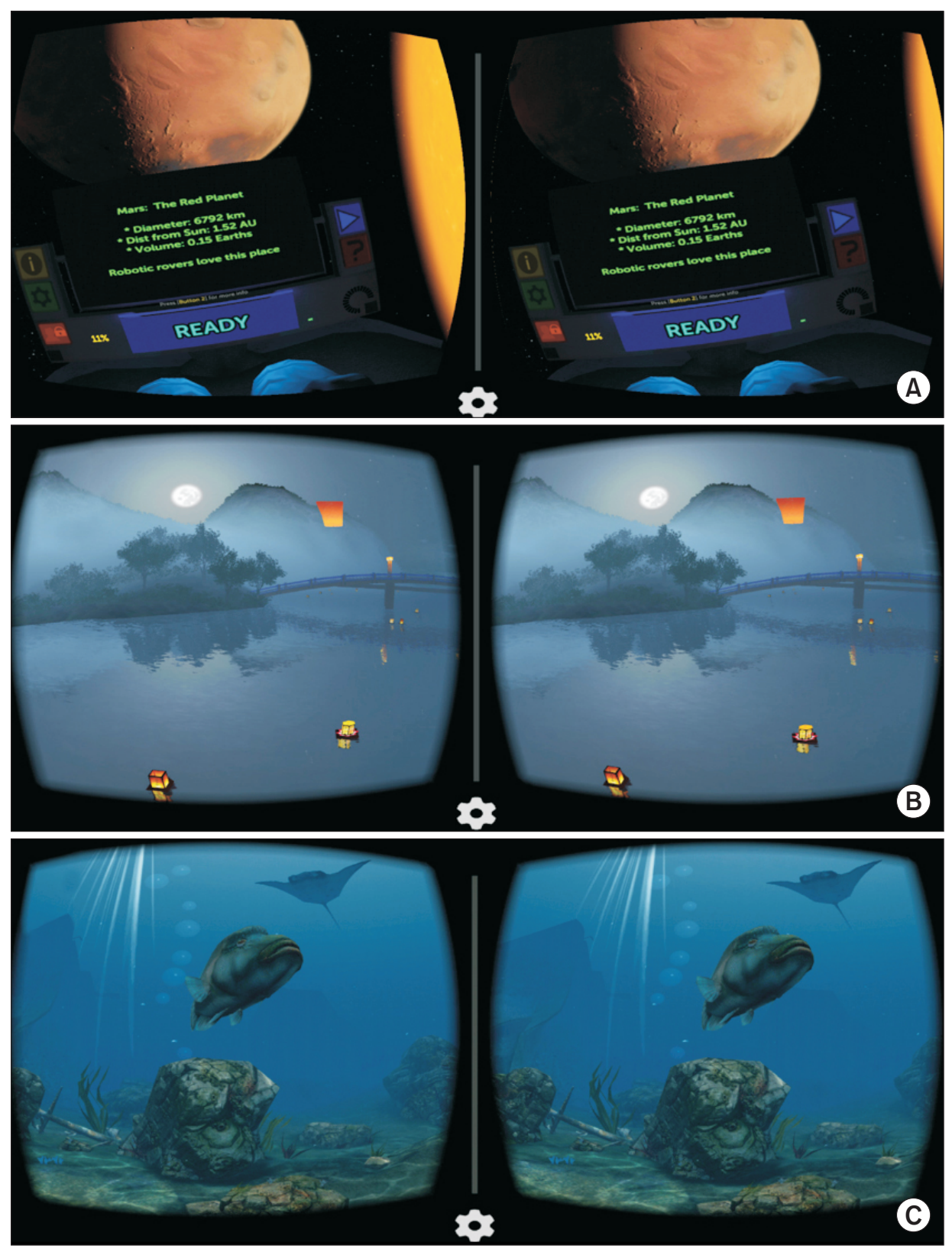

Fig. 2. Representative images of the three free virtual reality applications available to patients: (A) Titans of Space (Drash VR LLC, Murrieta, CA, USA), (B) Lanterns for Google Cardboard (Oleksandr Popov, Svitlovodsk, Ukraine), and (C) SeaWorld VR2 (eiNpictures, Incheon, South Korea).

the next. SeaWorld is designed as an underwater scuba dive; users observe ocean life underwater by looking around. Lanterns is the least interactive and represents a night view during a lantern festival; flickering paper lanterns are seen in the air and on water with bridges, trees, and mountains in the background.

\section{Nerve block procedure}

All patients received an ACC using a standardized technique, as described previously [10]. Briefly, after peripheral intravenous catheter insertion in the preoperative block room and positioning, standard noninvasive monitors and oxygen, via a nasal cannula, were applied to all patients. After performing a timeout procedure, patients received intravenous sedation at the discretion of the regional anesthesiologist (if not offered VR) or used VR with intravenous sedation upon request. Each patient using VR was asked by the regional anesthesiologist and VR coach during the perineural catheter insertion procedure if they were experiencing any pain, and, if so, whether they would like to receive intravenous pain medication. All procedures were performed by a regional anesthesiology and acute pain medicine fellow under direct supervision by an attending regional anesthesiologist. Under ultrasound guidance (Edge HFL50x Transducer; Fujifilm Sonosite, Bothell, WA, USA) and 
using sterile technique, each ACC (Arrow FlexTip Plus; Teleflex Medical, Research Triangle Park, NC, USA) was inserted through a local anesthetic skin wheal and directed in-plane into the adductor canal lateral to the superficial femoral artery after an injection of $10-20 \mathrm{ml}$ of $1.5 \%$ mepivacaine via the placement needle. All catheters were tunneled subcutaneously in a cephalad and lateral direction towards the anterior superior iliac spine using the placement needle and its stylet [10]. The catheter was secured with a clear occlusive dressing and an anchoring device [11]. Patients' procedure-related pain scores (numeric rating scale [NRS], 0-10: $0=$ no pain, $10=$ worst pain) were obtained and recorded at the conclusion of catheter placement, tunneling, and dressing application. Each patient received direct and uninterrupted monitoring by a physician anesthesiologist or nurse practitioner on the regional anesthesiology and acute pain medicine team from the time of perineural catheter insertion until the patient was transported to the operating room by the intraoperative anesthesiologist.

\section{Outcomes}

The primary outcome was fentanyl dosage $(\mu \mathrm{g})$. Secondary outcomes were midazolam dosage $(\mathrm{mg})$, procedure-related pain (NRS 0-10), procedural time (min), and blood pressure change scores (mmHg; highest systolic and diastolic blood pressure recordings minus baseline pre-procedural systolic and diastolic blood pressure recordings).

\section{Statistical analysis}

Statistical analyses were performed with NCSS-PASS software (Kaysville, UT, USA). Normality of distribution was determined using the Kolmogorov-Smirnov test. Single comparisons of continuous data were performed using Student's $t$-test if normally distributed; the Mann-Whitney $U$ test was used for continuous data with non-normal distributions. The $\mathrm{Z}$ test or Fisher's exact test ( $\mathrm{n}<5$ in any field) were used for comparisons of categorical data. A two-sided $\mathrm{P}<0.05$ was considered to indicate statistical significance for the primary outcome.

\section{Results}

Over the course of 1 month, fourteen sequential patients underwent elective unilateral primary TKA and received a preoperative ACC; seven patients received usual care without VR, and seven were offered and chose to try VR. The baseline characteristics between groups were similar except for height (Table 1). Five of the seven VR patients used Titans of Space throughout perineural catheter insertion. One chose Lanterns from the start because he wanted something more relaxing. One patient started with Titans of Space but had difficulty with gaze fixation due to his position (laying flat) and was changed to SeaWorld by the coach.

\section{Primary outcome}

The primary outcome, fentanyl dose, was lower (median [10th-90th percentiles]) in the group that used VR (0 [0-20] $\mu \mathrm{g}$ ) versus the non-VR group (50 [30-100] $\mu \mathrm{g} ; \mathrm{P}=0.008)$. Of the seven patients who used VR, only one (14\%) received intravenous sedation (fentanyl alone) versus six of seven (86\%) who received usual care $(\mathrm{P}=0.029)$; one patient in the non-VR group requested no intravenous sedation.

\section{Secondary outcomes}

Midazolam use was lower in the VR group (0 [0-0] $\mathrm{mg}$ ) compared with the non-VR group $(1[0-1] \mathrm{mg} ; \mathrm{P}=0.024)$. The

Table 1. Baseline Characteristics

\begin{tabular}{lccr}
\hline & $\begin{array}{c}\text { Non-VR } \\
(\mathrm{n}=7)\end{array}$ & $\begin{array}{c}\text { VR } \\
(\mathrm{n}=7)\end{array}$ & P value \\
\hline Age $(\mathrm{yr})$ & $67(58-78)$ & $69(61-71)$ & 0.806 \\
Sex $(\mathrm{F} / \mathrm{M})$ & $0 / 7$ & $0 / 7$ & $>0.999$ \\
ASA Physical Status & $3(2-3)$ & $3(3-3)$ & 0.530 \\
Height $(\mathrm{cm})$ & $174(168-179)$ & $180(175-188)$ & 0.044 \\
Weight $(\mathrm{kg})$ & $114(84-131)$ & $103(93-144)$ & 0.543 \\
Body mass index $\left(\mathrm{kg} / \mathrm{m}^{2}\right)$ & $40(29-41)$ & $35(28-45)$ & $>0.999$ \\
Preoperative opioid use & 2 & 2 & $>0.999$ \\
History of mental illness & 1 & 4 & 0.266 \\
Systolic blood pressure (mmHg) & $140(127-156)$ & $147(130-161)$ & 0.804 \\
Diastolic blood pressure (mmHg) & $76(69-88)$ & $81(62-89)$ & 0.849 \\
\hline
\end{tabular}

Values are reported as the median (10th-90th percentiles) or number of subjects (n), as indicated. VA: virtual reality, ASA: American Society of Anesthesiologists. 
Table 2. Secondary Outcomes

\begin{tabular}{lccc}
\hline & $\begin{array}{c}\text { Non-VR } \\
(\mathrm{n}=7)\end{array}$ & $\begin{array}{c}\text { VR } \\
(\mathrm{n}=7)\end{array}$ & P value \\
\hline Procedural duration $(\mathrm{min})$ & $20(15,32)$ & $20(15,32)$ & $>0.999$ \\
Change in systolic blood pressure $(\mathrm{mmHg})$ & $2(-2,12)$ & $5(2,12)$ & 0.453 \\
Change in diastolic blood pressure $(\mathrm{mmHg})$ & $0(-4,5)$ & $5(-8,13)$ & 0.583 \\
\hline
\end{tabular}

Values are reported as the median (10th-90th percentiles).

one patient in the VR group who received intravenous sedation answered "yes" when he was asked if he was feeling pain during the perineural catheter insertion procedure, replied affirmatively when offered the option of receiving intravenous pain medication, and was given a one-time intravenous bolus of fentanyl (50 $\mu \mathrm{g})$. Patients in the non-VR group received intravenous sedation at the discretion of the regional anesthesiologist as a bolus at the start of the perineural catheter insertion procedure. Of these six patients, four received both midazolam and fentanyl, and two received fentanyl alone. Procedure-related pain was lower in the VR group (1 [1-4] NRS) versus the non-VR group (3 [2-6] NRS; $\mathrm{P}=0.032$ ). There was no difference in other outcomes (Table 2). No patients who received VR reported dizziness or experienced nausea, vomiting, or other undesirable effects.

\section{Discussion}

The use of VR distraction during preoperative ultrasoundguided ACC insertion for patients scheduled to undergo TKA nearly eliminated the need for intravenous sedation and reduced procedure-related pain without increasing the procedural duration. VR distraction has no known long-lasting side effects, unlike opioids, and limiting opioid exposure may affect patients' development of opioid-induced hyperalgesia in the perioperative period [12]. Given the prevalence of long-term opioid use after even low-risk surgery $[13,14]$, anesthesiologists need to explore non-opioid analgesic modalities to maximize patient comfort and safety.

VR devices, once very expensive, are now available commercially at low cost and can be used in conjunction with many smartphones. Free applications are available for download from application stores of major phone and tablet manufacturers, making this technology readily accessible. VR originates from the gaming industry, and there has been growing interest in its use in other fields, especially healthcare. VR has previously been described alone or as an adjunct to reduce acute pain and anxiety related to burn wound care [15-18], periodontal procedures [19], and needle-related procedures involving pediatric cancer patients [20]. VR is designed to allow patients to escape to a computer-generated virtual world away from the hospital setting in which they are undergoing painful procedures. The posi- tive effects of VR on pain experience are attributed to humans' limited attentional capacity; because pain perception during a procedure requires dedicated attention, VR distracts attention away from the painful stimulus and immerses the patient in the virtual environment instead [18].

In the outpatient surgery setting, VR on mobile phones has been shown to provide anxiolysis during procedures performed under local or regional anesthesia [21]. VR distraction has not been previously studied as a method to prevent procedure-related pain in the field of anesthesiology, and the present report is the first to describe its use during perineural catheter insertion as an alternative to intravenous opioids and other sedatives. Our findings build on previous work in the area of VR distraction for acute pain management. In particular, our experience most closely resembles the results described by previous investigators who have studied the use of VR distraction for procedures in burned and wounded soldiers [16-18]. Like burn debridements and dressing changes, the perineural catheter insertion procedure represents a relatively brief but acutely painful stimulus. Additionally, compared with the active military participants included in previous studies involving burn victims [16-18], our population of veterans is likely to suffer from similar mental health issues, including post-traumatic stress disorder and anxiety, which can influence the pain experience.

There are several limitations to this retrospective study. First, this was designed as a QI project in the context of clinical care and not a randomized clinical trial. Consequently, there was no blinding or an assigned control group, no placebo, and no other form of distraction. Given the small scope of this project, our findings should be considered preliminary. Our results with VR distraction cannot be applied to every peripheral nerve block site; we chose one procedure location and patient population specifically because patient movement during the VR experience was not expected to be problematic. We also used a standardized ultrasound-guided perineural catheter insertion technique that is regularly practiced at our institution. This would not apply to nerve blocks of the upper extremity or neuraxial procedures during which VR-induced movement may create a patient safety risk. Our results may also not be reproducible if using a different technique for ACC insertion or different perineural catheter equipment. The intervention was dependent on one portable 
VR device and the availability of a separate practitioner, uninvolved in direct patient care, to provide coaching on the use of VR during preoperative perineural catheter insertion in a block room. We acknowledge that these resources may not be available at every institution.

In summary, the use of VR distraction during preoperative ACC insertion decreased the use of intravenous opioids and sedatives and reduced procedure-related pain without increasing the procedural duration. Our data indicate that VR distraction may provide an effective nonpharmacological alternative to intravenous sedation for the ultrasound-guided placement of certain perineural catheters. Future studies to explore other potential applications of VR distraction in the perioperative period are warranted.

\section{Conflict of Interest}

Dr. Mariano has received unrestricted educational program funding paid to his institution from Halyard Health (Alpharetta, GA, USA) and B Braun (Bethlehem, PA, USA). These companies had no input into any aspect of the present study design and implementation; data collection, analysis and interpretation; or manuscript preparation.

\section{ORCID}

T. Edward Kim, https://orcid.org/0000-0002-1183-3556

Edward R. Mariano, https://orcid.org/0000-0003-2735-248X

\section{References}

1. Mariano ER, Loland VJ, Sandhu NS, Bellars RH, Bishop ML, Afra R, et al. Ultrasound guidance versus electrical stimulation for femoral perineural catheter insertion. J Ultrasound Med 2009; 28: 1453-60.

2. Mariano ER, Cheng GS, Choy LP, Loland VJ, Bellars RH, Sandhu NS, et al. Electrical stimulation versus ultrasound guidance for poplitealsciatic perineural catheter insertion: a randomized controlled trial. Reg Anesth Pain Med 2009; 34: 480-5.

3. Kim TE, Howard SK, Funck N, Harrison TK, Walters TL, Wagner MJ, et al. A randomized comparison of long-axis and short-axis imaging for in-plane ultrasound-guided popliteal-sciatic perineural catheter insertion. J Anesth 2014; 28: 854-60.

4. Mulier JP. Perioperative opioids aggravate obstructive breathing in sleep apnea syndrome: mechanisms and alternative anesthesia strategies. Curr Opin Anaesthesiol 2016; 29: 129-33.

5. Sun EC, Darnall BD, Baker LC, Mackey S. Incidence of and risk factors for chronic opioid use among opioid-naive patients in the postoperative period. JAMA Intern Med 2016; 176: 1286-93.

6. Guenther U, Riedel L, Radtke FM. Patients prone for postoperative delirium: preoperative assessment, perioperative prophylaxis, postoperative treatment. Curr Opin Anaesthesiol 2016; 29: 384-90.

7. Malloy KM, Milling LS. The effectiveness of virtual reality distraction for pain reduction: a systematic review. Clin Psychol Rev 2010; 30 : 1011-8.

8. Das DA, Grimmer KA, Sparnon AL, McRae SE, Thomas BH. The efficacy of playing a virtual reality game in modulating pain for children with acute burn injuries: a randomized controlled trial [ISRCTN87413556]. BMC Pediatr 2005; 5: 1.

9. Webb CA, Mariano ER. Best multimodal analgesic protocol for total knee arthroplasty. Pain Manag 2015; 5: 185-96.

10. Mariano ER, Kim TE, Wagner MJ, Funck N, Harrison TK, Walters T, et al. A randomized comparison of proximal and distal ultrasoundguided adductor canal catheter insertion sites for knee arthroplasty. J Ultrasound Med 2014; 33: 1653-62.

11. Borg L, Howard SK, Kim TE, Steffel L, Shum C, Mariano ER. A comparison of strength for two continuous peripheral nerve block catheter dressings. Korean J Anesthesiol 2016; 69: 506-9.

12. Mauermann E, Filitz J, Dolder P, Rentsch KM, Bandschapp O, Ruppen W. Does fentanyl lead to opioid-induced hyperalgesia in healthy volunteers?: a double-blind, randomized, crossover trial. Anesthesiology 2016; 124: 453-63.

13. Alam A, Gomes T, Zheng H, Mamdani MM, Juurlink DN, Bell CM. Long-term analgesic use after low-risk surgery: a retrospective cohort study. Arch Intern Med 2012; 172: 425-30.

14. Rozet I, Nishio I, Robbertze R, Rotter D, Chansky H, Hernandez AV. Prolonged opioid use after knee arthroscopy in military veterans. Anesth Analg 2014; 119: 454-9.

15. Morris LD, Louw QA, Grimmer-Somers K. The effectiveness of virtual reality on reducing pain and anxiety in burn injury patients: a systematic review. Clin J Pain 2009; 25: 815-26.

16. Maani CV, Hoffman HG, Morrow M, Maiers A, Gaylord K, McGhee LL, et al. Virtual reality pain control during burn wound debridement of combat-related burn injuries using robot-like arm mounted VR goggles. J Trauma 2011; 71(1 Suppl): S125-30.

17. Maani CV, Hoffman HG, Fowler M, Maiers AJ, Gaylord KM, Desocio PA. Combining ketamine and virtual reality pain control during severe burn wound care: one military and one civilian patient. Pain Med 2011; 12: 673-8.

18. Hoffman HG, Chambers GT, Meyer WJ 3rd, Arceneaux LL, Russell WJ, Seibel EJ, et al. Virtual reality as an adjunctive non-pharmacologic analgesic for acute burn pain during medical procedures. Ann Behav Med 2011; 41: 183-91.

19. Furman E, Jasinevicius TR, Bissada NF, Victoroff KZ, Skillicorn R, Buchner M. Virtual reality distraction for pain control during 
periodontal scaling and root planing procedures. J Am Dent Assoc 2009; 140: 1508-16.

20. Nilsson S, Finnström B, Kokinsky E, Enskär K. The use of Virtual Reality for needle-related procedural pain and distress in children and adolescents in a paediatric oncology unit. Eur J Oncol Nurs 2009; 13: 102-9.

21. Mosso JL, Gorini A, De La Cerda G, Obrador T, Almazan A, Mosso D, et al. Virtual reality on mobile phones to reduce anxiety in outpatient surgery. Stud Health Technol Inform 2009; 142: 195-200. 